\title{
Prevalência dos transtornos mentais na população adulta brasileira: uma revisão sistemática de 1997 a 2009
}

Prevalence of mental disorders in the Brazilian adult population: a systematic review from 1997 to 2009

Élem Guimarães dos Santos', Marluce Miguel de Siqueira²

\section{RESUMO}

Introdução: Esta revisão sistemática de literatura se propõe a verificar os índices de prevalência dos transtornos mentais na população adulta brasileira. Métodos: Foram pesquisadas as bases de dados Medline e Lilacs, no período de 1997 a 2009, utilizando os seguintes descritores: "transtornos mentais", "estudos de prevalência", "Brasil" e seus correspondentes em inglês "mental disorders", "cross-sectional studies", "Brazil". Foram excluídos os artigos: sem resumo/abstract, sem descrição de estudo tipo transversal, sem descrição ou referência a estimativas que verificassem a prevalência de transtornos mentais, estudos com populações específicas, estudos com crianças, adolescentes e idosos e, ainda, os estudos escritos em outros idiomas diferentes do português, inglês e espanhol. Esses critérios resultaram na seleção de 25 artigos. Resultados: Nos estudos analisados foram encontrados altos índices de prevalência geral de transtornos mentais na população adulta. Esses índices variaram entre 20\% e 56\%, acometendo principalmente muIheres e trabalhadores. Discussão: Os estudos analisados nesta revisão mostraram um panorama geral dos transtornos mentais na população adulta brasileira, descrevendo como se dá a sua distribuição nessa população, apontando para taxas elevadas, muitas vezes associadas a sexo, idade, condições socioeconômicas, condições de trabalho e estilos de vida dos indivíduos.

\section{ABSTRACT}

Introduction: This systematic review of literature aims to verify prevalence rates of mental disorders in the Brazilian adult population. Methods: We searched the Medline and Lilacs in the period from 1997 to 2009 using the following descriptors: "mental disorders", "cross-section studies", "Brazil". Exclusion criteria of articles were: no abstract, no description of cross-sectional study, did not contain the estimate that would verify the prevalence of mental disorders, studies with specific populations and further studies with children, adolescents and elderly, and written in other languages other than Portuguese, English and Spanish. These criteria resulted in the selection of 25 articles. Results: In the studies reviewed found high rates of overall prevalence of mental disorders in adults. These rates varied between 20\% and 56\%, affecting mainly women and workers. Discussion: The studies analyzed in this review show an overview of mental disorders in Brazilian adult population, describing how is their distribution in this population, pointing to high rates, often associated with sex, age, socioeconomic, work conditions and lifestyles of individuals.

1 Universidade Federal do Espírito Santo (UFES), Programa de Pós-Graduação em Saúde Coletiva. Núcleo de Estudos sobre Álcool e outras Drogas, Centro de Ciências da Saúde (NEAD-CCS), UFES. 


\section{INTRODUÇÃO}

Segundo a Classificação Internacional de Transtornos Mentais e de Comportamento (CID-10)', os transtornos mentais (TM) se classificam como doença com manifestação psicológica associada a algum comprometimento funcional resultante de disfunção biológica, social, psicológica, genética, física ou química. Podem ser classificados, ainda, como alterações do modo de pensar e/ou do humor associadas a uma angústia expressiva, produzindo prejuízos no desempenho global da pessoa no âmbito pessoal, social, ocupacional e familiar.

Frequentemente encontrados na comunidade, os TM geram alto custo social e econômico; são universais, pois atingem pessoas de todas as idades, causando incapacitações graves e definitivas que elevam a demanda nos serviços de saúde. Mas o ônus dos TM foi subestimado durante muito tempo, principalmente porque a forma de avaliar seu impacto na saúde valorizava apenas os índices de mortalidade. Os TM assumem valores baixos de mortalidade, mas possuem, todavia, um grande peso de incapacidade de duração longa, acarretando a redução da qualidade de vida dos indivíduos.

Os TM só foram reconhecidos como um sério problema de saúde pública a partir de 1996, quando pesquisadores da Universidade de Harvard e da Organização Mundial de Saúde (OMS) publicaram um estudo ${ }^{2}$ utilizando como medida a combinação do número de anos vividos com a incapacidade e o número de anos perdidos por morte prematura causada pela doença (que tem como indicador os DALY - disability adjusted life of years -, ou anos de vida perdidos ajustados por incapacidade), obtendo resultados que confirmam esse reconhecimento. Nesse estudo, das 10 principais causas de incapacitação em todo o mundo, cinco delas estavam associadas aos TM, entre elas a depressão (13\%), a ingestão de álcool (7,1\%), os distúrbios afetivos bipolares (3,3\%), a esquizofrenia (4\%) e os distúrbios obsessivo-compulsivos (2,8\%).

O relatório da OMS e da Organização Pan-Americana de Saúde (OPAS) ${ }^{3}$ assinala que os TM correspondem a 12\% da carga mundial de doenças e a $1 \%$ da mortalidade, quando menos de $1 \%$ dos recursos da saúde é investido em ações para a saúde mental. Apesar disso, mais de $40 \%$ dos países ainda carecem de políticas em saúde mental e 30\% não têm programas nessa esfera. Sabe-se ainda que a maioria dos transtornos é tratável e evitável, corroborando a premissa de que, quando se investe na prevenção e promoção da saúde mental, se pode reduzir bastante o número de incapacidades resultantes desses transtornos.

Um estudo realizado pelo Consórcio Internacional de Epidemiologia Psiquiátrica (ICPE) da OMS revelou que, dentre os países da América Latina, o Brasil apresentou a maior prevalência de TM na população adulta de 15-59 anos ${ }^{4}$, com índices elevados para os transtornos da ansiedade, transtornos do humor e os transtornos relacionados ao uso de substâncias psicoativas.
Dados do Ministério da Saúde apontam que 3\% da população geral brasileira sofrem com TM graves e persistentes, $6 \%$ apresentam transtornos psiquiátricos graves decorrentes do uso de álcool e outras drogas e 12\% necessitam de algum atendimento, seja ele contínuo ou eventual. O índice de utilização dos serviços de saúde ainda é baixo, está em torno de $13 \%$. A maioria das pessoas com algum transtorno mental não busca atendimento psiquiátrico, por razões que estão ligadas, muitas vezes, ao estigma, ao desconhecimento da doença, ao preconceito, à falta de treinamento das equipes para lidar com esses transtornos, à falta de serviços adequados para atendimento psiquiátrico, ao medo, entre outras ${ }^{5,6}$.

No Brasil, ainda não se tem um estudo representativo dos índices de prevalência de indivíduos afetados pelos TM, mas uma estimativa da extensão do problema encontrada em alguns estudos. Os estudos epidemiológicos são de grande importância para determinar essa magnitude, sendo muito úteis e relevantes nas decisões e no planejamento de políticas públicas de saúde mental, na organização dos serviços e no desenvolvimento de programas de prevenção e tratamento?.

Nesse sentido, esta revisão sistemática de literatura se propõe a verificar os índices de prevalência dos transtornos mentais na população adulta brasileira.

\section{MÉTODO}

\section{Estratégia de busca}

Foram revisados artigos publicados de 1997 a 2009, que avaliaram os índices de prevalência de TM na população adulta brasileira. Foram pesquisadas as bases de dados Medline e Lilacs utilizando os seguintes descritores: "transtornos mentais", "estudos de prevalência", "Brasil" e seus correspondentes em inglês "mental disorders", "cross-sectional studies", "Brazil".

\section{Identificação e triagem}

Os critérios de exclusão/inclusão adotados nesta revisão foram baseados em estudos populacionais realizados no Brasil $^{8}$. Foram excluídos os artigos sem resumo/abstract, sem descrição de estudo tipo transversal, sem descrição ou referência a estimativas que verificassem a prevalência de TM, descrição de estudos com populações específicas, estudos com crianças, adolescentes e idosos*, e escritos em outros idiomas diferentes do português, inglês e espanhol.

Foram localizados na base de dados Lilacs 26 artigos: 6 eram estudos com populações específicas**, 4 estudos incluíam crianças e adolescentes ou idosos na amostra e em

\footnotetext{
* Foram excluídos estudos com crianças e adolescentes menores de 15 anos e idosos com 60 anos ou mais. Essa faixa etária foi excluída pelo fato de a maioria dos estudos com adultos incluir populações entre 15 e 59 anos. ** Populações específicas excluídas: pacientes, portadores de TM específicos, pacientes com HIV, com hepatite B ou C, gestantes, população de rua/albergues, portadores de alguma deficiência ou doença autoimune.
} 
10 estudos não havia descrição ou associação com o estimador de interesse, pois se tratavam de artigos sobre indicadores demográficos, reestruturação produtiva, acesso a medicamentos, validação de questionário e associação entre transtornos específicos. Restaram 6 artigos para leitura.

Na base de dados Medline (1997-2009), com os termos em português, foram encontrados 58 artigos: 17 já haviam sidos descritos no Lilacs (repetidos), 9 artigos eram com populações específicas, 4 estudos com crianças e adolescentes ou idosos, 1 artigo sem resumo, 1 estudo de caso-controle e 9 artigos sem associação ou referência ao estimador de interesse, por se tratarem de artigos sobre avaliação de autonomia, protocolo de estudo, ajustamento social, transtornos específicos, padrões de consumo de psicofármacos, validação de questionário e análise de gênero. Restaram 17 artigos. Na segunda busca, com os termos em inglês, foram localizados 109 artigos. Desses, 52 artigos já haviam sido citados no Medline em português, 18 artigos eram sobre populações específicas, 15 artigos descreviam estudos com crianças e adolescentes ou idosos, 1 estava sem resumo, 2 estudos foram feitos fora do Brasil e 19 artigos não possuíam refe- rências de associação entre as estimativas de interesse, pois se tratavam dos mesmos assuntos relatados anteriormente. Restaram 2 artigos.

Grande parte dos artigos estava disponível no portal online da CAPES: http://periodicos.capes.gov.br. Daqueles que não estavam disponibilizados no portal, foram solicitadas cópias diretamente para os autores ou pelo Serviço Cooperativo de Acesso a Documentos (SCAD).

No total, restaram 25 artigos com texto completo para avaliar a elegibilidade nesta revisão. Abaixo é descrito o fluxograma de todo o processo de identificação e seleção dos artigos.

\section{Estratégia de análise e seleção}

\section{Critérios de elegibilidade}

Os critérios de elegibilidade foram baseados em estudos populacionais realizados no Brasil ${ }^{8}$. Nesse sentido, foram incluídos os estudos com perda amostral inferior a 20\%, estudos que referiram estimativas de prevalência para problemas de saúde mental em população de 15 a 59 anos e estudos que utilizaram instrumentos padronizados de avaliação psiquiátrica para a coleta dos dados. Foram ainda observados, com relação à
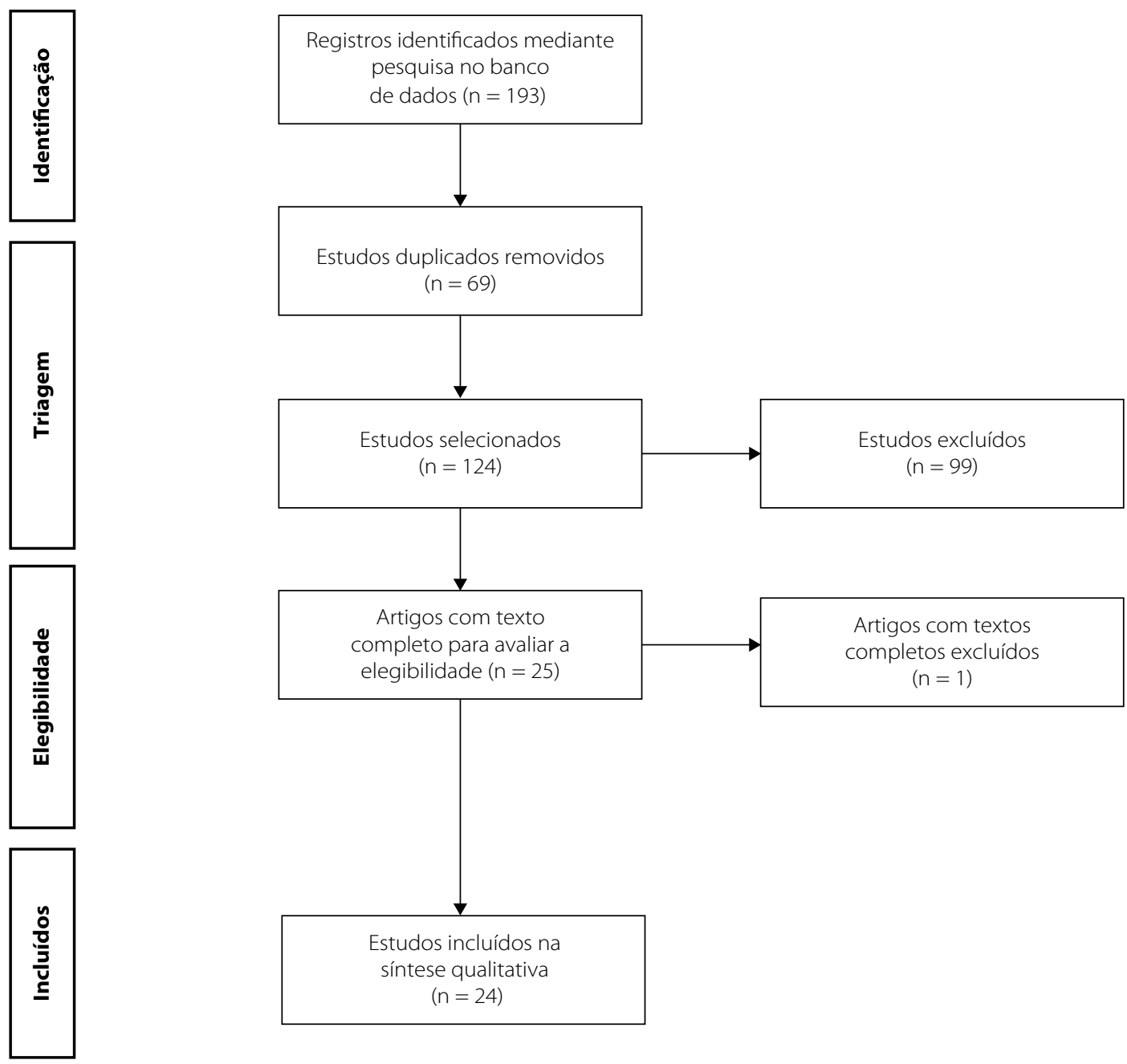

Fluxograma 1. Processo de identificação e seleção dos artigos.

Fonte: Moher et al. ${ }^{7}$ 
elegibilidade dos estudos, o método de amostragem utilizado (amostragem probabilística), o risco de viés e as medidas tomadas para evitar esse erro, principalmente no processo de seleção da amostra ou na obtenção das informações dos estudos, tendo em vista a possibilidade de erro dos estudos transversais.

Dentre os instrumentos utilizados nos estudos analisados nesta revisão, para detecção dos sintomas psiquiátricos, estão os questionários autoaplicáveis, método de investigação rápido e de fácil administração. Entre eles, o Self Report Questionnaire (SRQ-20), questionário desenvolvido pela OMS, para uso em estudos em países em desenvolvimento e para identificação de possíveis casos de TM. É composto por 20 questões com respostas de "sim" ou "não" sobre sintomas emocionais e físicos associados a quadros psiquiátricos. Em estudos realizados no Brasil9,10, estabeleceu-se como ponto de corte para os casos suspeitos de TM 7/8 ou mais respostas positivas. Mas a efetividade do instrumento tende a variar com a amostragem e as características culturais da população em estudo. Alguns dos estudos que serão aqui apresentados utilizaram outros escores, baseando-se na entrevista psiquiátrica como padrão-ouro para redefinição do ponto de corte. Outro instrumento muito utilizado em estudos transversais é o General Health Questionnaire (GHQ-12)1", validado no Brasil por Mari e Williams ${ }^{12}$ e concebido como método de rastreamento de TM na saúde clínica geral. É composto por 12 questões, sendo considerados suspeitos indivíduos com escore igual ou maior que 4 (ponto de corte $3 / 4$ ).

O Questionário de Morbidade Psiquiátrica em Adultos (QMPA) é outro instrumento muito utilizado em estudos de base populacional, validado e desenvolvido principalmente para populações urbanas brasileiras ${ }^{13,14}$. Possui 44 itens de triagem para detecção de TM em adultos, sendo considerados positivos os indivíduos que apresentarem escore maior que 7. Outro tipo de instrumento utilizado em estudos seccionais é a versão brasileira do Composite International Diagnostic Interview $(\mathrm{CIDI})^{15}$, entrevista psiquiátrica estruturada, desenvolvida pela OMS para ser usada por entrevistadores leigos na detecção de casos positivos de TM.

A maioria desses instrumentos busca apenas apontar problemas na função mental, deixando a pesquisa diagnóstica para uma abordagem posterior, pois detectam apenas os TM leves, também chamados de distúrbios psiquiátricos menores (DPM) ou transtornos mentais comuns (TMC). Os TM leves são aqueles relacionados aos transtornos de ansiedade, depressão, transtornos somatoformes e as neuroses. Transtornos graves como as psicoses, manias, esquizofrenias não são detectados nesses instrumentos, por necessitarem de uma avaliação psiquiátrica mais apurada.

Após análise dos artigos quanto à elegibilidade dos estudos, foi excluído o estudo de Vedovato e Monteiro ${ }^{16}$, que não explicitou detalhes referentes ao instrumento utilizado, não se referindo ao método de identificação e diagnóstico dos casos para os achados de prevalência, obtendo ainda uma perda de seguimento superior a $20 \%$.

A tabela 1 mostra a relação dos estudos selecionados para análise qualitativa, agrupados conforme população estudada, método de amostragem, perda amostral, método de identificação dos TM e a prevalência encontrada.

\section{RESULTADOS}

Poucos são os estudos que tentaram sumarizar dados de prevalências dos TM em adultos. No Brasil, Mello et al. ${ }^{8}$ apresentam, em seu livro Epidemiologia da saúde mental no Brasil, alguns estudos populacionais realizados com a população brasileira. Na América Latina, vale destacar o estudo de Kohn et al. ${ }^{17}$, que reuniu várias publicações da América Latina e do Caribe, entre 1980 e 2004, apontando para a necessidade de se conhecer a prevalência dos transtornos mentais, a carga de morbidade, a redução da qualidade de vida que estes geram na população e a lacuna no tratamento, dada pela diferença entre as taxas de prevalência verdadeiras e as taxas de pessoas tratadas nos serviços de saúde, colocando os transtornos psiquiátricos como assunto prioritário para a saúde pública e a necessidade da ampliação dos programas, atualização e formulação de novas políticas de saúde mental. Nesse estudo, as psicoses afetivas e a esquizofrenia foram observadas em 1,4\% da população em algum momento da vida, sem diferenças significantes entre os sexos; a depressão mostrou uma prevalência de 8,7\% na vida, 4,9\% no ano e 4,3\% no mês, acompanhada dos transtornos da ansiedade, acometendo principalmente as mulheres; os transtornos relacionados ao uso de substâncias psicoativas tiveram uma prevalência de 11,3\% no ano para o uso, abuso e dependência do álcool e 0,5\% para o abuso de outras drogas, afetando principalmente homens. Entre os estudos brasileiros incluídos na revisão de Kohn et al. ${ }^{17}$, está o de Vorcaro et al. ${ }^{18}$, que determinou a prevalência de depressão numa comunidade pequena (Bambuí/MG), com adultos maiores de 18 anos, apontando altas taxas de prevalência de depressão na vida $(15,6 \%)$, no ano $(10,0 \%)$ e no mês (8,2\%). Um importante estudo populacional realizado no Brasil, descrito por Kohn et al. ${ }^{17}$, Mello et al. ${ }^{8}$ e encontrado nesta revisão sistemática, foi o de Almeida Filho et al. ${ }^{19}$, um dos pioneiros no país que avaliou a prevalência de TM em 6.476 indivíduos maiores de 15 anos. Foi um estudo multicêntrico realizado em três grandes centros urbanos brasileiros (São Paulo, Porto Alegre e Brasília), que ocorreu em dois estágios: o primeiro estágio foi a aplicação do QMPA para detectar os casos suspeitos de TM; o segundo consistiu na entrevista psiquiátrica dos casos positivos para confirmação da presença de morbidade psiquiátrica, utilizando-se o inventário de sintomas do Manual de Diagnóstico e Estatística de Distúrbios Mentais (DSM-III). Os índices de prevalência de TM para toda a vida foram de 
Tabela 1. Relação dos estudos transversais incluídos e excluídos da análise

\begin{tabular}{|c|c|c|c|c|c|}
\hline Artigo & Método de amostragem & Perda amostral & População do estudo & $\begin{array}{l}\text { Método de } \\
\text { identificação } \\
\text { dos TM } \\
\end{array}$ & Prevalência de TM \\
\hline \multicolumn{6}{|l|}{ Estudos na comunidade } \\
\hline Almeida-Filho et al., $1997^{19}$ & Amostragem aleatória estratificada & Inferior a $20 \%$ & $\begin{array}{l}6.476 \text { indivíduos com pelo menos } \\
15 \text { anos }\end{array}$ & QMPA & Prevalência total: $20 \%$ a $35 \%$ \\
\hline Andrade et al., $2002^{20}$ & Amostragem aleatória estratificada & Inferior a $20 \%$ & 1.464 indivíduos maiores de 18 anos & CIDI & $\begin{array}{l}\text { Prevalência de TM na vida: } 45,9 \% \\
\text { Prevalência de TM no ano: } 26,8 \% \\
\text { Prevalência de TM no mês: } 22,2 \%\end{array}$ \\
\hline De Lima et al., $1999^{21}$ & Amostragem aleatória simples & Inferior a $20 \%$ & 1.277 pessoas com 15 anos ou mais & SRQ-20 & Prevalência de TM total: 22,7\% \\
\hline $\begin{array}{l}\text { Ludemir, } 2000^{22} \\
\text { Ludemir e Lewis, } 2001^{23} \\
\text { Ludemir e Lewis, } 2003^{24} \\
\text { Ludemir e Lewis, } 2005^{25} \\
\text { Ludemir e Melo-Filho, } 2002^{26}\end{array}$ & Amostragem aleatória simples & Inferior a $20 \%$ & 621 pessoas de 15 anos ou mais & SRQ-20 & Prevalência de TMC: 35\% \\
\hline Costa e Ludemir, $2005^{27}$ & Amostragem sistemática & Inferior a $20 \%$ & 483 indivíduos maiores de 19 anos & SRQ-20 & Prevalência de TMC: 36\% \\
\hline Coelho et al., $2009^{28}$ & Amostragem aleatória simples & Inferior a $20 \%$ & 1.327 indivíduos de 40 anos ou mais & SRQ-20 & Prevalência de TMC: $30,2 \%$ \\
\hline \multicolumn{6}{|l|}{ Estudos com professores } \\
\hline Delcor et al., $2004^{29}$ & Amostragem aleatória simples & Inferior a $20 \%$ & $\begin{array}{l}250 \text { professores } \\
\text { Média de idade: } 34,5 \text { anos } \\
(D P=7,5 \text { anos })\end{array}$ & SRQ-20 & Prevalência de DPM:41,5\% \\
\hline Reis et al., $2005^{30}$ & Amostragem sistemática & Inferior a $20 \%$ & 808 professores & SRQ-20 & $\begin{array}{l}\text { Prevalência de DPM: } 55,9 \% \\
\text { professores }\end{array}$ \\
\hline Gasparini et al., $2006^{31}$ & Amostragem sistemática & Inferior a $20 \%$ & 751 professores & GHQ-12 & Prevalência de TMC: $50,3 \%$ \\
\hline Vedovato e Monteiro, $2008^{16}$ & Amostragem não probabilística & Superior a $20 \%$ & 468 professores & Nenhum & Prevalência de TM: 20,9\% \\
\hline \multicolumn{6}{|l|}{ Estudo com trabalhadores } \\
\hline Silva e Menezes, $2008^{32}$ & Amostragem sistemática & Inferior a $20 \%$ & $141 \mathrm{ACS}$ & SRQ-20 & Prevalência de TMC geral: 43,3\% \\
\hline Souza e Silva, $1998^{34}$ & Amostragem aleatória simples & Inferior a $20 \%$ & $\begin{array}{l}925 \text { motoristas e cobradores de } \\
\text { ônibus }\end{array}$ & SRQ-20 & Prevalência de DPM: 20,3\% \\
\hline Faria et al., $1999^{35}$ & Amostragem aleatória simples & Inferior a $20 \%$ & 1.282 trabalhadores rurais & SRQ-20 & Prevalência geral de DPM: $38 \%$ \\
\hline Faria et al., $2000^{36}$ & Amostragem aleatória simples & Inferior a $20 \%$ & 1.479 trabalhadores rurais & SRQ-20 & Prevalência geral de TMC: 36\% \\
\hline Veggi et al., $2004^{37}$ & Amostragem sistemática & Inferior a $20 \%$ & $\begin{array}{l}3.526 \text { funcionários de uma } \\
\text { universidade do RJ } \\
\text { Idade: } 22-59 \text { anos }\end{array}$ & GHQ-12 & $\begin{array}{l}\text { Prevalência de TMC geral: } \\
34,5 \% \text { mulheres } \\
22,3 \% \text { homens }\end{array}$ \\
\hline Pinho e Araújo, $2007^{33}$ & Amostragem sistemática & Inferior a $20 \%$ & 80 trabalhadores de enfermagem & SRQ-20 & Prevalência de TM: 26,3\% \\
\hline \multicolumn{6}{|l|}{ Estudo com estudantes } \\
\hline Loayza et al., $2001^{38}$ & Amostragem aleatória simples & Inferior a $20 \%$ & 302 estudantes & SRQ-20 & Prevalência de TM: 22,9\% \\
\hline Facundes e Ludemir, $2005^{39}$ & Amostragem aleatória simples & Inferior a $20 \%$ & $\begin{array}{l}461 \text { alunos graduandos da } \\
\text { Universidade de Pernambuco }\end{array}$ & SRQ-20 & Prevalência de TMC geral: 34,1\% \\
\hline \multicolumn{6}{|l|}{ Estudo com mulheres } \\
\hline Santana et al., $1997^{40}$ & Amostragem aleatória estratificada & Inferior a $20 \%$ & $\begin{array}{l}327 \text { mulheres trabalhadoras } \\
\text { formais e informais }\end{array}$ & QMPA & Prevalência de TM: 28,1\% \\
\hline Araújo et al., $2003^{41}$ & Amostragem sistemática & Inferior a $20 \%$ & $\begin{array}{l}502 \text { mulheres trabalhadoras de } \\
\text { enfermagem entre } 30 \text { e } 49 \text { anos }\end{array}$ & SRQ-20 & Prevalência de TM: 33,3\% \\
\hline Araújo et al., $2006^{42}$ & Amostragem aleatória estratificada & Inferior a $20 \%$ & $\begin{array}{l}1.235 \text { mulheres com } 15 \text { anos ou } \\
\text { mais }\end{array}$ & SRQ-20 & $\begin{array}{l}\text { Prevalência de TMC: } \\
\text { Donas de casa: } 43,5 \% \\
\text { Trabalhadoras: } 36,8 \%\end{array}$ \\
\hline
\end{tabular}

51\% em Brasília, 43\% em Porto Alegre e 30\% em São Paulo. Entre as mulheres, foram encontradas prevalências maiores em Porto Alegre (50\%) e Brasília (54\%), enquanto em São Paulo a prevalência foi maior nos homens (33\%). Os índices de prevalência no ano variaram entre 19\% e 34\%, sendo maiores em Brasília e Porto Alegre. Com relação às prevalências específicas por diagnósticos, tanto as estimativas de TM na vida ou no ano, o transtorno de ansiedade apareceu em primeiro lugar, seguido dos estados fóbicos e depressivos e o uso/abuso e dependência do álcool. O conjunto de distúrbios delirantes (esquizofrenias, manias e outros quadros psicóticos) atingiu prevalências que variaram entre 1\% e 4\%.
A distribuição da prevalência global de TM de acordo com o gênero revelou que o alcoolismo constituiu o principal problema de saúde mental no grupo masculino, com prevalências em torno de 15\% nas três amostras. Nas mulheres, houve predomínio dos transtornos da ansiedade, estados fóbicos, depressões e transtornos somatoformes.

Outro importante estudo analisado na revisão de Kohn et al. ${ }^{17}$ e Mello et al. ${ }^{8}$, incluído nesta revisão, está o de Andrade et al. ${ }^{20}$, que obtiveram dados sobre a prevalência de TM na população com 18 anos ou mais, residente numa área de captação de um grande complexo hospitalar na cidade de São Paulo. Utilizando como instrumento o CIDI, com uma 
amostra de 1.464 pessoas, foram obtidas prevalências elevadas na comunidade, similares a de estudos realizados em outros países. As prevalências de TM na vida, no ano e no mês foram, respectivamente, de 45,9\%, 26,8\% e 22,2\%. Os TM mais prevalentes (na vida, no ano e no mês, respectivamente) foram: dependência do tabaco $(25 \%, 11,4 \%, 9,3 \%)$, transtornos do humor $(18,5 \%, 7,6 \%, 5 \%)$, transtornos do humor com episódios depressivos (16,8\%, 7,1\%, 4,5\%), transtornos de ansiedade $(12,5 \%, 7,7 \%, 6 \%)$, transtornos somatoformes (6\%, 4,2\%, 3,2\%) e abuso/dependência de álcool (5,5\%, 4,5\%, 4\%). Não foram encontradas diferenças de gênero na morbidade global. No entanto, as mulheres apresentaram maior risco para os transtornos não psicóticos, quando excluídos os transtornos relacionados ao uso de substâncias psicoativas, mais prevalentes na população masculina.

Nesta revisão, foram encontrados outros estudos que avaliaram os índices de prevalência de TM com amostra domiciliar em adultos na comunidade brasileira. Entre eles, está o de De Lima et al. ${ }^{21}$, realizado com 1.277 pessoas com 15 anos ou mais, em domicílios da região de Pelotas, RS, que corrobora com os resultados de estudos realizados fora do país. Esse estudo utilizou o SRQ-20 como instrumento e obteve uma prevalência geral de $22,7 \%$. Segundo o sexo, a prevalência foi maior entre as mulheres (26,5\%) em comparação aos homens (17,9\%). A prevalência de ingestão alcoólica em nível de risco foi maior entre os homens $(11,9 \%)$, enquanto nas mulheres esse índice foi de 4,1\%.

Nos estudos de Ludemir ${ }^{22}$, Ludemir e Lewis ${ }^{23-25}$ e Ludemir e Melo-Filho ${ }^{26}$, verificaram-se as condições socioeconômicas e sua influência na ocorrência dos TMC. Nesses estudos, foi utilizada uma mesma amostra de 226 domicílios, onde foram entrevistadas 621 pessoas maiores de 15 anos por meio do SRQ-20 e, posteriormente, a entrevista psiquiátrica foi feita para confirmação dos casos baseada no DSM-III. Esses estudos, respectivamente citados, investigaram a associação entre desemprego e o trabalho informal e a ocorrência dos TMC; associação entre condições socioeconômicas e os TMC; associação entre trabalho informal e os TMC; diferenças entre gêneros e a associação com trabalho informal e TMC. A prevalência geral de TMC nesses estudos foi de $35 \%{ }^{26}$, enquanto a prevalência por sexo foi de $20,7 \%$ para os homens e 45,4\% para as mulheres ${ }^{20,23}$. Em relação à faixa etária, a prevalência foi maior em pessoas com idade acima de 55 anos (47,9\%), seguidas da faixa etária de 25-54 anos (36,1\%) e 15$24 \operatorname{anos}(25,7 \%)^{20,23}$.

O estudo de Costa e Ludemir ${ }^{27}$, conduzido na Zona da Mata em Pernambuco, com uma população de 483 moradores da comunidade de Pirauá, maiores de 19 anos, investigou a prevalência dos TMC e sua associação com o apoio social, obtendo uma prevalência geral de $36 \%$, sendo na faixa etária entre $20-39$ anos (27,2\%), entre $40-59$ anos $(43,3 \%)$ e 60 anos ou mais (38,8\%). A prevalência por sexo foi de $24,5 \%$ em homens e $44 \%$ em mulheres. A maior prevalência foi em mulheres de 40-59 anos, em pessoas analfabetas, divorciadas, separadas ou viúvas e indivíduos com renda per capita abaixo de 50 reais.

O estudo de base populacional de Coelho et al. ${ }^{28}$ avaliou a prevalência de TMC em pessoas com mais de 40 anos numa área urbana de Pelotas, RS. Nesse estudo, foram entrevistados 1.327 indivíduos, utilizando o SRQ-20 para detecção de casos suspeitos de distúrbios psiquiátricos. A prevalência de TMC foi de $30,2 \%$, sem alguma diferença significativa entre os sexos. As taxas foram mais elevadas em indivíduos divorciados, desempregados, com menor escolaridade e entre 46-55 anos.

Alguns estudos encontrados investigaram a ocorrência de TM na população de professores. O estudo de Delcor et al. ${ }^{29}$ descreve as condições de trabalho e saúde de 250 professores da rede de ensino particular em Vitória da Conquista, BA, utilizando o SRQ-20 como instrumento para detectar possíveis suspeitas de DPM. Do total da amostra, 82,8\% eram mulheres, com média de idade de 34,5 anos (desvio-padrão - DP $=7,5$ anos), $65,1 \%$, casadas e $72,1 \%$ com nível de escolaridade superior. A prevalência de DPM foi de $41,5 \%$. Nesse estudo, utilizou-se, ainda, um questionário de avaliação para abuso ou dependência de álcool, o CAGE (acrônimo referente às suas quatro perguntas - Cut down, Annoyed by criticism, Guilty e Eye-opener), que encontrou 22,1\% de consumo de bebidas alcoólicas entre os professores e, desses, 1,3\% foi classificado como dependente. Além disso, o hábito de fumar foi referido por 4,2\% e eram ex-fumantes 11,4\%.

No estudo de Reis et al..$^{30}$, com 808 professores da rede municipal de Vitória da Conquista, BA, em sua maioria mulheres $(94,1 \%)$, casada $(52,3 \%)$, com filhos $(58,5 \%)$ e com escolaridade de nível médio (67,5\%), média de idade de 34,2 anos (DP $=8,5)$, utilizando o SRQ-20 para avaliação psiquiátrica, foi encontrada prevalência geral de distúrbios psíquicos de $55,9 \%$, sendo $57,2 \%$ nas mulheres e $34 \%$ nos homens. Além disso, o consumo de bebidas alcoólicas foi referido por $22 \%$ dos entrevistados e o hábito de fumar, por 7,3\%. A frequência de respostas positivas para as perguntas do SRQ-20 foi mais alta nas questões relacionadas ao humor depressivo/ ansioso, à diminuição da energia e aos sintomas somáticos.

O estudo de Gasparini et al. ${ }^{31}$ estimou a prevalência de TMC em 751 professores da rede municipal de ensino de Belo Horizonte, MG, associando às características do trabaIho. Utilizou como instrumento o GHQ. A maioria dos entrevistados foi de mulheres $(55,5 \%)$, e a média de idade dos entrevistados foi de 41 anos (DP $=8), 55,5 \%$ deles eram casados e $92,9 \%$ tinham pelo menos um curso superior. Foi encontrada uma prevalência de TMC de 50,3\%, sendo 91,1\% para as mulheres e $8,9 \%$ para os homens. A prevalência foi maior na faixa etária entre 40-49 anos (40,2\%), seguida de 30-39 anos (37,8\%). O consumo de bebidas alcoólicas foi referido por $67 \%$ da amostra, 9,8\% eram fumantes e 9,7\% utilizavam algum medicamento para dormir. 
Entre outros estudos com grupos de trabalhadores, encontra-se o estudo de Silva e Menezes ${ }^{32}$ realizado com trabaIhadores da saúde, especificamente os agentes comunitários de saúde (ACS), que utilizou o SRQ-20 para estimar a prevalência de esgotamento profissional e os TMC, numa amostra de 141 ACS, em sua maioria mulheres (92,2\%), com média de idade de 38,9 anos ( $(\mathrm{DP}=11,4)$, predomínio da faixa etária de 31-40 anos, com ensino fundamental ou médio completo (73\%). Foi encontrada uma prevalência geral de $43,4 \%$ entre os ACS, sendo o risco maior entre as mulheres. Além disso, foi referido o uso regular de antidepressivos por $17 \%$ dos ACS. O estudo de Pinho e Araújo ${ }^{33} \mathrm{com} 80$ trabalhadores de enfermagem, em sua maioria mulheres acima de 36 anos, utilizando o SRQ-20 para casos suspeitos de TM, revelou uma prevalência de 26,3\%, que foi associada à idade (acima de 36 anos), estado civil (solteira), nível superior de escolaridade e renda de até três salários-mínimos.

No estudo de Souza e Silva ${ }^{34}$, utilizando uma amostra de 925 motoristas e cobradores de ônibus e o SRQ-20 como instrumento de avaliação psiquiátrica, observou-se uma prevalência de 20,3\%: 13\% entre cobradores e 28\% entre motoristas. Houve associação significativa com a faixa etária (maior prevalência entre menores de 30 anos), salário mensal, tempo de residência na região metropolitana de São Paulo e a procedência e as condições relativas ao trabalho (tipo de trânsito, regulagem de banco, tempo de trabalho, alteração da escala e absenteísmo).

Sobre os trabalhadores rurais, foram encontrados dois estudos realizados na Serra Gaúcha, RS, que avaliaram a prevalência de distúrbios psiquiátricos e as condições do modo de produção do trabalho rural. O primeiro estudo foi o de Faria e outros (1999) ${ }^{35}$, que utilizou uma amostra de 1.282 trabalhadores maiores de 15 anos, pertencentes à família proprietária de 446 estabelecimentos. Nessa amostra, a maioria era de homens (55\%), com média de idade de 42,2 anos (DP $=15,7 \%)$ e média de escolaridade de 4,8 anos ( $\mathrm{DP}=2,7)$. Relativamente aos agricultores, 38\% apresentaram casos suspeitos para DPM com base no SRQ-20. Além disso, 19\% haviam usado remédios psiquiátricos e $5 \%$ já haviam sido hospitalizados por problemas psiquiátricos em algum momento da vida. Utilizando-se o CAGE para avaliação do abuso ou dependência de álcool, $6 \%$ dos trabalhadores foram avaliados como "bebedor-problema". No estudo de Faria et al. ${ }^{36}$ de 2000, na mesma localidade, utilizando um tamanho de amostra diferente da do estudo anteriormente citado, com 1.479 trabalhadores rurais de 495 propriedades, em sua maioria mulheres, com idade média de 41,4 anos ( $D P=15,6)$ e de escolaridade de 4,8 anos ( $D P=2,7$ ), observou-se uma prevalência de DPM de $36 \%$, com predomínio na faixa etária entre 50-59 anos. Houve maior prevalência no sexo feminino (42,5\%) em relação ao sexo masculino (31,6\%). Além disso, $7 \%$ apresentaram teste CAGE positivo, 13\% eram fumantes e $12 \%$, ex-fumantes.
Um último estudo encontrado com a população de trabalhadores foi o de Veggi et al. ${ }^{37}$, conduzido com 3.526 funcionários de uma universidade do Rio de Janeiro, com idade entre 22 e 59 anos, utilizando o GHQ-12 para identificação de casos suspeitos de TMC e sua associação com a percepção inadequada de peso corporal. Foram encontradas prevalências de $34,5 \%$ entre as mulheres e $22,3 \%$ entre os homens. Maiores prevalências foram encontradas em mulheres com menor escolaridade e renda. Os problemas de saúde autorrelatados, tanto em homens como em mulheres, foram mais frequentes naqueles em que o GHQ-12 foi positivo.

Dois estudos transversais com uma população de estudantes foram encontrados nesta revisão. O primeiro foi o estudo de Loayza et al. ${ }^{38}$, que avaliou a associação entre insônia e teste positivo para TM utilizando o SRQ-20. Participaram do estudo 302 alunos, em sua maioria homens, com média de idade de 20,4 anos ( $D P=1,89$ ). A prevalência de casos positivos no SRQ foi de 22,1\%; desses, 38,8\% entre mulheres e 61,2\% entre homens. A associação entre insônia e SRQ positivo foi significante. O segundo estudo encontrado foi o de Facundes e Ludemir ${ }^{39}$, que determinou a prevalência de TMC na população de 461 estudantes de graduação da Universidade de Pernambuco, utilizando o SRQ-20. A maioria dos entrevistados foi de mulheres (69,8\%), entre $20-22$ anos (54,2\%). Em geral, a prevalência de TMC na população estudada foi de 34,1\%, com maior prevalência entre os estudantes de medicina $(42,6 \%)$ quando comparados com os alunos dos outros cursos: odontologia (33,3\%), educação física $(31,8 \%)$ e enfermagem (25\%).

Nesta revisão, foram encontrados três estudos transversais com populações de mulheres, especificamente. O estudo de Santana et al.40, utilizando como instrumento o QMPA, analisou o papel do trabalho informal na origem das disfunções mentais entre mulheres. $\mathrm{O}$ estudo foi conduzido com uma amostra de 327 mulheres trabalhadoras formais e informais. Dentre as mulheres trabalhadoras formais, $42 \%$ pertenciam à faixa etária de 26-49 anos; entre as trabalhadoras informais, prevaleceu a faixa etária acima de 39 anos. Observou-se prevalência geral de $28,1 \%$ de TM entre as mulheres, ocorrendo maiores índices entre as trabalhadoras informais.

No estudo de Araújo et al..$^{41}$ com 502 trabalhadoras de enfermagem entre 30 e 49 anos, que avaliou a associação entre o controle sobre o trabalho, demandas psicológicas e a ocorrência de TM, utilizando o SRQ-20, foi encontrada uma prevalência de 33,3\%. Houve forte associação entre demanda psicológica do trabalho e os distúrbios psíquicos, principalmente nos grupos em que as trabalhadoras estiveram expostas a fatores como alta demanda de trabalho $(56,1 \%)$, alta exigência no trabalho $(57,5 \%)$ e baixo controle no trabalho $(62,5 \%)$, sendo a alta exigência um importante preditor dos distúrbios psiquiátricos.

Araújo et al. ${ }^{42}$ publicaram, em 2006, outro estudo com mulheres que estimou a prevalência de TMC entre donas de casa e trabalhadoras, utilizando o SRQ-20. A população do 
estudo constitui-se de 1.235 mulheres com 15 anos ou mais. A prevalência de TMC entre as donas de casa foi de $43,5 \%$ e entre as trabalhadoras, de 36,8\%. Houve predominância dos sintomas psiquiátricos relacionados com o humor depressivo/ansioso (74,1\% das donas de casa e $66,6 \%$ das trabalhadoras), seguidos dos sintomas somáticos (49,3\% das donas de casa e 46,9\% das trabalhadoras). Mulheres acima de 40 anos, viúvas/separadas e analfabetas nos dois grupos apresentaram maiores prevalências de TMC.

\section{DISCUSSÃO}

Os estudos encontrados nesta revisão revelaram o panorama geral da ocorrência dos TMC na população adulta, apontando algumas diferenças em relação aos transtornos específicos. Em relação ao gênero, observou-se que as mulheres são mais acometidas pelos transtornos de ansiedade, de humor e os somatoformes, enquanto nos homens há uma prevalência dos transtornos relacionados ao uso de substâncias psicoativas. Muitos estudos ${ }^{43,44}$ tentam explicar essa diferença, associando a ocorrência desses transtornos específicos nas mulheres a fatores hormonais e psicológicos. Outra explicação é que as mulheres teriam maior facilidade de identificar os sintomas, admiti-los e buscar ajuda, enquanto os homens tendem a buscar nas substâncias psicoativas o alívio para seu sofrimento ou angústia. O fato de a maioria dos entrevistados nas pesquisas ser de mulheres também seria outro motivo para o aumento dos índices de prevalência nesse grupo, não sendo possível fazer muitas inferências ao grupo masculino; além disso, o instrumento utilizado na pesquisa poderia influenciar nesses índices, pois a maioria dos questionários autoaplicáveis refere-se a questões relacionadas ao humor, à ansiedade ou às fobias e detecta transtornos relativamente mais frequentes em mulheres.

Tomando-se a idade como referência, indivíduos na faixa etária de 25-54 anos apresentam maiores índices de TM. Alguns fatores relacionados às condições socioeconômicas, como o desemprego, a baixa escolaridade, o estado civil (divorciado, separado ou viúvo), o sexo, as condições precárias de habitação, o trabalho informal e o não acesso aos bens de consumo, foram identificados como possíveis determinantes para os altos índices de TM nos estudos analisados.

Relativamente aos trabalhadores, entre eles, professores, trabalhadores rurais, enfermeiros, ACS, motoristas e cobradores, foram encontrados elevados índices de prevalência dos TM. A rotina de trabalho, a demanda, a exigência, o controle, o processo de trabalho e as condições ambientais foram associados ao aparecimento desses distúrbios.

A combinação das altas taxas de prevalência, o início cada vez mais precoce, a redução da qualidade de vida e o comprometimento no desempenho global da pessoa (pessoal, familiar, ocupacional, emocional e social) fazem com que os
TM sejam um dos principais contribuintes para a carga de doença tota ${ }^{45}$. É notável a grande sobrecarga de incapacidade que os TM causam na qualidade de vida das pessoas. No Brasil e no mundo, com o processo de transição epidemiológica, a carga de doenças relacionadas aos agravos não transmissíveis tem-se elevado rapidamente e sua prevenção tem sido o maior desafio para saúde pública ${ }^{46}$. No campo dos DALY, as doenças neuropsiquiátricas ocupam a primeira posição, e os TM englobam 10,8\% nesse campo para ambos os sexos ${ }^{46}$.

Atrelado a isso, observa-se que em muitos países não há políticas de saúde mental e outros ainda estão em fase de implementação dos serviços de atenção comunitária. No Brasil, isso se inicia por volta da década de 1990, paralelamente à implantação do Sistema Único de Saúde (SUS). Apesar disso, novos medicamentos, terapias e intervenções psicossociais vêm se intensificando no tratamento dos transtornos psiquiátricos. Mas ainda há um grande impasse no tratamento, principalmente em relação ao acesso e à procura pelos serviços de saúde para o tratamento. Existem barreiras culturais, financeiras e estruturais que impedem o acesso e a procura pelo serviço, que estão atreladas a muitos fatores como o estigma ou desconhecimento da doença, a percepção de ineficácia do tratamento, a pouca disponibilidade de serviços, a falta de treinamento das equipes da atenção básica para a identificação dos casos, entre outros.

Outras implicações se referem às limitações do tipo de estudo. A grande maioria dos estudos é de corte transversal e os resultados são difíceis de interpretar por causa dos problemas metodológicos como a falta de padronização e rigor na definição e identificação de casos, amostragem pouco estruturada, risco de viés, imprecisão e precariedade na definição e mensuração dos fatores de exposição e de confusão relevantes, além das limitações intrínsecas dos estudos de prevalência, que permitem apenas descrever a ocorrência, a distribuição e as características dos TM numa determinada população, não dando margem para inferir causalidade.

Nesse sentido, são necessários mais estudos analíticos (coorte) para conhecer melhor os fatores de risco, o prognóstico, a distribuição e a frequência dos TM na população e, ainda, o impacto que eles geram na qualidade de vida das pessoas. Apesar de ser difícil medir toda a gama de sofrimento e incapacidade que os TM geram, a associação das entrevistas psiquiátricas a instrumentos que possam medir a qualidade de vida ou o impacto dos sintomas dos TM sobre a vida da população deve ser incluída em novas pesquisas. Além disso, novos estudos devem dimensionar a morbidade psiquiátrica no âmbito do acesso aos serviços de saúde e às intervenções terapêuticas, avaliando sua efetividade e os fatores que interferem nesse processo.

\section{AGRADECIMENTOS}

Ao Núcleo de Estudos sobre Álcool e outras Drogas (NEAD) da Universidade Federal do Espírito Santo (UFES), pelo apoio 
técnico e científico. À Lívia Nossa, aluna de iniciação científica do NEAD, pela revisão do abstract. Ao professor Edson Theodoro, pelo apoio prestado nesta revisão.

Não existem conflitos de interesse, incluindo interesses financeiros e relações e filiações específicas relevantes ao tema deste artigo.

\section{REFERÊNCIAS}

1. Organização Mundial da Saúde. Classificação de TM e de comportamento da CID-10. Porto Alegre: Artes Médicas; 1993.

2. Lopez AD, Murray CC. The global burden of disease, 1990-2020. Nat Med. 1998:4(11):1241-3.

3. Organização Pan-Americana de Saúde. Organização Mundial da Saúde. Relatório sobre a saúde no mundo 2001. Saúde Mental: nova concepção, nova esperança. OMS; 2001.

4. World Health Organization. Cross-national comparisons of the prevalences and correlates of mental disorders. Bulletin WH0. 2000;78(4):413-26.

5. Brasil. Ministério da Saúde. Secretaria de Atenção à Saúde. Departamento de Ações Programáticas e Estratégicas. Coordenação Geral de Saúde Mental. Saúde Mental em Dados. Ano II, n. 4, agosto de 2007.

6. Mari JJ, Jorge MR, Kohn R. Epidemiologia dos transtornos psiquiátricos em adultos. In: Mello MF, Mello, AAF, Kohn R, organizadores. Epidemiologia da saúde mental no Brasil. Porto Alegre: Artmed; 2007, p. 119-41.

7. Moher D, Liberati A, Tetzlaff J, Altman DG, et al. Preferred reporting items for systematic reviews and meta-analyses: The PRISMA statement. PLoS Med. 2009;6(7):e1000097.

8. Mello MF, Mello AAF, Kohn R, orgs. Epidemiologia da saúde mental no Brasil. Porto Alegre: Artmed; 2007.

9. Mari JJ. A validity study of a Psychiatric Screening Questionnaire (SRQ-20) in primary care in the city of São Paulo. Br J Psychiatric. 1986;148:23-6.

10. Fernandes SRP, Almeida Filho N. Validação do SRQ em amostra de trabalhadores em informática. Rev Bras Saude Ocup. 1998;89:105-12.

11. Goldberg DP, Gater R, Sartorius N, Usrun TB, Piccinelli M, Gureje 0 . The validity of two versions of the GHQ in the WHO study of mental illness in general health care. Psychol Med. 1997:27:191-7.

12. Mari JJ, Williams P. A comparison of the validity of two psychiatric screening questionnaires in Brazil, using ROC analysis. Psychol Med. 1985;15:651-9.

13. Santana V. Estudo epidemiológico das doenças mentais em um bairro em Salvador. Série de Estudos em Saúde. 1982;3(Supl 2):122.

14. Almeida Filho N, Mari JJ, Coutinho E, et al. Estudo multicêntrico de morbidade mental psiquiátrica em áreas urbanas brasileiras (Braślila, São Paulo e Porto Alegre). Revista ABP/ APAL. 1992;14:93-104.

15. Robins LN, Wing J, Wittchen HU, Helzer JE, Babor TF, Burke J, et al. The composite International Diagnostic Interview: an epidemiologic instrument suitable for use in conjunction with different diagnostic systems and in different cultures. Arch Gen Psychiatry. 1988:45:1069-77.

16. Vedovato TG, Monteiro MI. Perfil sociodemográfico e condições de saúde e trabalho dos professores de nove escolas estaduais paulistas. Rev Esc Enferm USP. 2008;42(2);290-7.

17. Kohn R, Levav I, De Almeida JM, Vicente B, Andrade L, Caraveo-Anduaga JJ, et al. Mental disorders in Latin America and the Caribbean: a public health priority. Rev Panam Salud Publica. 2005:18(4-5):229-40.

18. Vorcaro CM, Lima-Costa MF, Barreto SM, Uchoa E. Unexpected high prevalence of 1-month depression ins a small Brazilian community: the Bambuí Study. Acta Psychiatr Scand. 2001;104:257-63.

19. Almeida-Filho N, Mari JJ, Coutinho E, França JF, Fernandes J, Andreoli SB, et al. Brazilian multicentric study of psychiatric morbidity: methodological features and prevalence estimates. Br J Psychiatric. 1997;171:524-9.

20. Andrade L, Walters EF, Gentil V, Laurenti R. Prevalence of ICD-10 mental disorders in a catchment area in the city of São Paulo, Brazil. Soc Psychiatry Psychiatr Epidemiol. 2002:37:316-25
21. De Lima MS, Hotopt M, Mari JJ, Béria JU, De Bastos AB, Mann A. Psychiatric disorder and the use of benzodiazepines: in example of the inverse care law from Brazil. Soc Psychiatry Psychiatr Epidemiol. 1999;34:316-22.

22. Ludemir AB. Inserção produtiva, gênero e saúde mental. Cad Saude Publica. 2000;16(3):647-59.

23. Ludemir AB, Lewis $G$. Links between social class and common mental disorders in Northeast Brazil. Soc Psychiatry Psychiatr Epidemiol. 2001;36:101-7.

24. Ludemir $A B$, Lewis G. Informal work and common mental disorders. Soc Psychiatry Psychiatr Epidemiol. 2003;38:485-9.

25. Ludermir $A B$, Lewis $G$. Is there a gender difference on the association between informal work and common mental disorders? Soc Psychiatry Psychiatr Epidemiol. 2005;40:622-7.

26. Ludemir AB, Mello-Filho DA. Condições de vida e estrutura ocupacional associadas a transtornos mentais comuns. Rev Saude Publica. 2002;36(2):213-21.

27. Costa AG, Ludemir AB. Transtornos mentais em comunidade rural da Zona da Mata de Pernambuco, Brasil. Cad Saude Publica. 2005;21(1):73-9.

28. Coelho FMC, Pinheiro RT, Horta BL, Magalhães PVS, Garcias CMM, Silva CV. Common mental disorders and chronic non-communicable diseases in adults: a population-based study. Cad Saude Publica. 2009;25(1):59-67.

29. Delcor NS, Araújo TM, Reis EJFB, Porto LA, Carvalho FM, Silva M0, et al. Condições de trabalho e saúde dos professores da rede particular de ensino de Vitória da Conquista, Bahia, Brasil. Cad Saude Publica. 2004;20(1):187-96.

30. Reis EJFB, Carvalho FM, Araújo TM, Porto LA, Silvany Neto AM. Trabalho e distúrbios psíquicos em professores da rede municipal de Vitória da Conquista, Bahia, Brasil. Cad Saude Publica. 2005;21(5):1480-90

31. Gasparini SM, Barreto SM, Assunção AA. Prevalência de transtornos mentais comuns em professores da rede municipal de Belo Horizonte, Minas Gerais, Brasil. Cad Saude Publica. 2006;22(12):2679-91.

32. Silva ATC, Menezes PR. Esgotamento profissional e transtornos mentais comuns em agentes comunitários de saúde. Rev Saude Publica. 2008:42(5):921-9.

33. Pinho OS, Araújo TM. Trabalho de enfermagem em uma unidade de emergência hospitalar e transtornos mentais. R Enferm UERJ. 2007;15(3):329-36.

34. Souza MFM, Silva GR. Risco de distúrbios psiquiátricos menores em área metropolitana na região Sudeste do Brasil. Rev Saude Publica. 1998;32(1):50-8.

35. Faria NMX, Facchini LA, Fassa AG, Tomasi E. Estudo transversal sobre saúde mental de agricultores da Serra Gaúcha (Brasil). Rev Saude Publica. 1999;33(4):391-400.

36. Faria NMX, Facchini LA, Fassa AG, Tomasi E. Processo de produção rural e saúde na Serra Gaúcha: um estudo descritivo. Cad Saude Publica. 2000;16(1):115-28.

37. Veggi AB, Lopes $C S$, Faerstein E, Sichieri R. Índice de massa corporal, percepção de peso corporal e transtornos mentais comuns entre funcionários de uma universidade no Rio de Janeiro. Rev Bras Psiquiatr. 2004;26(4):242-7.

38. Loayza MPH, Ponte TS, Carvalho CG, Pedrotti MR, Nunes PV, Souza CM, et al. Association between mental health screening by self-report questionnaire and insomnia in medical students. Arq Neuropsiquiatr. 2001;59(2-A):180-5.

39. Facundes VLD, Ludemir AB. Common mental disorders among health care students. Rev Bras Psiquiatr. 2005;27(3):194-200.

40. Santana VS, Loomis D, Newman B, Harlow SD. Informal jobs: another occupational hazard for women's mental health? Int J Epidemiol. 1997;26(6):1236-42.

41. Araújo TM, Aquino E, Menezes G, Santos C0, Aguiar L. Aspectos psicossociais do trabalho e distúrbios psíquicos entre trabalhadores de enfermagem. Rev Saude Publica. 2003;37(4):424-33.

42. Araújo TM,AlmeidaMMG, Santana CC, Araújo EM,Pinho PS. Transtornos mentais comuns em mulheres: estudo comparativo entre donas de casa e trabalhadoras. Rev Enferm UERJ. 2006;14(2):260-9.

43. Andrade LHSG, Viana MC, Silveira CM. Epidemiologia dos transtornos psiquiátricos na muIher. Rev Psiq Clín. 2006;33(2):43-54.

44. Kessler RC. Lifetime and 12-month prevalence of DSM-II-R psychiatric disorders in the United States: results from the national Comorbidity Survey. Arch Gen Psychiatry. 1994;51:8-19.

45. Hyman S, Chisholm D, Kessler R, Patel V, Whiteford H. Mental disorders. In: Jamison DT, Breman JG, Measham AR, Alleype G, Claeson M, Evans DB, et al. Disease Control Priorities in Developing Countries. The World Bank and the World Health Organization; 2006, p. 605-25.

46. Schramm JMA, Oliveira AF, Leite IC, Valente JG, Gadelha AMJ, Portela MC, et al. Transição epidemiológica e o estudo de carga de doença no Brasil. Cien Saude Colet. 2004;9(4):897-908. 